\title{
Use of tenofovir disoproxil fumarate is associated with a lower risk of hepatocellular carcinoma than entecavir in patients with chronic hepatitis B
}

\author{
Won-Mook Choi, Jonggi Choi \\ Department of Gastroenterology, Liver Center, Asan Medical Center, University of College of Medicine, Seoul 05505, Korea. \\ Correspondence to: Prof. Jonggi Choi, Department of Gastroenterology, Liver Center, Asan Medical Center, University of College \\ of Medicine, 88, Olympic-ro 43-gil, Songpa-gu, Seoul 05505, Korea. E-mail: j.choi@amc.seoul.kr
}

How to cite this article: Choi WM, Choi J. Use of tenofovir disoproxil fumarate is associated with a lower risk of hepatocellular carcinoma than entecavir in patients with chronic hepatitis B. Hepatoma Res 2021;7:74. https://dx.doi.org/10.20517/23945079.2021 .113

Received: 21 Aug 2021 First Decision: 13 Sep 2021 Revised: 18 Sep 2021 Accepted: 21 Oct 2021 Published: 5 Nov 2021

Academic Editor: Guang-Wen Cao Copy Editor: Yue-Yue Zhang Production Editor: Yue-Yue Zhang

\begin{abstract}
In patients with chronic hepatitis B (CHB), entecavir (ETV) and tenofovir disoproxil fumarate (TDF) are equally recommended as first-line treatment by the international guidelines. These two drugs have shown similar short and intermediate clinical outcomes, including virologic, biochemical, and histologic responses. However, there is considerable controversy as to whether ETV and TDF differ in reducing the risk of hepatocellular carcinoma (HCC) in patients with CHB despite many observational studies and meta-analyses being published. In this review, we summarize recent evidence comparing the preventive effects of these two drugs against HCC from the perspective that TDF is associated with a lower risk of HCC compared with ETV in patients with CHB.
\end{abstract}

Keywords: Hepatocellular carcinoma, hepatitis b virus, tenofovir disoproxil fumarate, entecavir, prevention

\section{INTRODUCTION}

Chronic hepatitis $\mathrm{B}(\mathrm{CHB})$ is one of the most common causes of chronic liver disease, with over 250 million people infected worldwide, accounting for $60 \%$ of hepatocellular carcinoma (HCC) cases in Asia and Africa and $20 \%$ of cases in the West ${ }^{[1-5]}$. Long-term nucleos(t)ide analog therapy reduces the risk of HCC by suppressing the replication of $\mathrm{HBV}$, a well-known risk factor for $\mathrm{HCC}^{[6,7]}$. Considering their high antiviral efficacy and low resistance rate, entecavir (ETV) and tenofovir-based regimens including tenofovir 
alafenamide and tenofovir disoproxil fumarate (TDF) have been equally recommended as first-line treatments for CHB by international guidelines ${ }^{[7-10]}$. ETV and TDF have shown comparable efficacy in viral suppression and biochemical response $e^{[9]}$. Both treatments have also been expected to be similarly effective in preventing the incidence of HCC, decompensation, and death ${ }^{[1,12]}$. Thus far, several observational studies have reported the incidence of HCC between the two treatments as secondary outcomes, not as a primary outcome for direct comparison. In 2018, Choi et al. ${ }^{[13]}$ reported that TDF treatment was associated with a lower risk of HCC compared with ETV in a nationwide cohort and hospital cohort study. Since then, several studies have shown the superiority of TDF over ETV in preventing HCC $^{[14,15]}$, whereas other studies failed to find a statistical difference between the two drugs ${ }^{[16-18]}$. This debate has been extended to the metaanalyses. Eleven meta-analyses were published between December 2019 and June 2021.

In this review, we summarize recent evidence comparing the effectiveness of these two treatments from the perspective that TDF may be superior to ETV in preventing HCC development.

\section{OBSERVATIONAL STUDIES}

\section{Studies showing the superiority of TDF in secondary prevention of HCC}

The study by Choi et al ${ }^{[13]}$ using the nationwide cohort and the large-scale single-center validation cohort was the first to find that TDF showed a significantly lower risk of HCC than ETV. This study of 24,156 and 2701 treatment-naive patients in the nationwide cohort and the hospital validation cohort showed that TDF conferred $34 \%$ and $32 \%$ reductions in the risk of HCC by multivariable analyses, respectively ${ }^{[13]}$. Of note, virological responses $[85.2 \%(\mathrm{TDF})$ vs. $78.7 \%(\mathrm{ETV}) ; P<0.001]$ and alanine aminotransferase (ALT) normalization rates $[44.3 \%(\mathrm{TDF}) v s .38 .7 \%(\mathrm{ETV}) ; P=0.002]$ at one year were significantly higher in TDF treatment compared with ETV treatment ${ }^{[13]}$.

Another study of 29,350 treatment-naïve patients with CHB from Hong Kong using a large administrative database demonstrated that TDF treatment showed a consistently lower risk of HCC [adjusted hazard ratio $(\mathrm{aHR})=0.36 ; 95 \%$ confidence interval $(\mathrm{CI}): 0.16-0.80 ; P=0.013]$ than ETV treatment across all of the sophisticated statistical analyses including multivariable, propensity score (PS)-matching, PS-weighting, and competing risk analyses. In addition, patients treated with TDF showed a significantly higher virological response at one year compared with those treated with ETV (77.6\% vs. 69.7\%, respectively $)^{[14]}$.

A multicenter retrospective study of 1560 cirrhotic patients from Taiwan reported that TDF significantly decreased the risk of HCC compared with ETV ( $\mathrm{aHR}=0.67 ; 95 \% \mathrm{CI}$ : 0.48-0.93; $P=0.002$ ), which was consistently observed in PS-matching analysis and PS-weighting analysis ${ }^{[19]}$. However, the lower risk of HCC in the TDF group was not observed in the subgroup analysis of patients with compensated cirrhosis at baseline to further exclude patients with decompensated cirrhosis or patients who were enrolled after 2011 to minimize the follow-up duration between the two groups ${ }^{[19]}$.

Another single-center study including 404 treatment-naïve patients with CHB from Korea observed that TDF treatment was associated with a significantly lower risk of HCC compared with ETV $(\mathrm{aHR}=0.31$; 95\%CI: 0.12-0.79; $P=0.014)$ in multivariable analysis, which was consistently observed in the PS-matching analysis ${ }^{[15]}$. In this study, statistical significance was not maintained when sustained virological suppression was adjusted in the PS-matching analysis ${ }^{[15]}$. However, it may not be statistically appropriate to include sustained virological response as a PS-matching variable because it cannot be measured at baseline.

Another western study presented at the International Digestive Disease Forum 2020 analyzed the United States administrative database, comparing ETV and TDF for the risk of HCC development in treatment- 
naïve patients with $\mathrm{CHB}^{[20]}$. In this study of 158,272 patients with $\mathrm{CHB}$, the absolute rate of HCC in the TDF group was approximately half that in ETV group. The lower risk of HCC in the TDF group compared with the ETV group $(\mathrm{aHR}=0.56 ; 95 \% \mathrm{CI}$ : 0.37-0.86) was persistently observed in multivariable analysis after adjustment for age group, sex, baseline health conditions, and PS weighting ${ }^{[20]}$.

\section{Studies showing the superiority of TDF in tertiary prevention of HCC}

Regarding the tertiary prevention of HCC after curative treatment, there are a few studies comparing the effectiveness of ETV and TDF in preventing HCC recurrence. A Chinese study including 233 patients with HBV-related HCC who underwent liver resections observed that TDF treatment was associated with a significantly longer disease-free survival compared with ETV (33 months vs. 24 months, respectively; $P<$ $0.001)^{[21]}$. Another study from China including 479 patients with HBV-related HCC confirmed this finding that TDF treatment was superior to non-TDF treatment including ETV $(\mathrm{aHR}=0.67 ; 95 \% \mathrm{CI}$ : 0.48-0.93; $P=$ 0.04) in preventing HCC recurrence after curative resection ${ }^{[22]}$.

Choi et al. ${ }^{[23]}$ conducted a retrospective single-center study of 1695 patients with HBV-related HCC with Barcelona Clinic Liver Cancer stage 0 or A who received curative resection, showing that TDF treatment was associated with a significantly lower risk of HCC recurrence ( $\mathrm{aHR}=0.82 ; 95 \% \mathrm{CI}: 0.68-0.98 ; P=0.03$ ) and death or liver transplantation (adjusted $\mathrm{HR}=0.62$; 95\%CI: 0.44-0.88; $P=0.01$ ) in multivariable analysis compared with ETV, which was maintained in PS-matching analysis. Intriguingly, the preventive effect of TDF was more prominent in preventing late recurrence $(\geq 2$ years after liver resection; $\mathrm{aHR}=0.68)$ than for preventing early recurrence $(<2 \text { years liver after resection; } \mathrm{aHR}=0.79)^{[23]}$, suggesting that TDF treatment might more effectively prevent the development of de novo HCC than ETV after curative resection ${ }^{[2,25]}$.

\section{META-ANALYSES}

In contrast to the above-mentioned studies, many studies failed to show a significant difference between the two drugs for preventing HCC occurrence ${ }^{[16-18,26-32]}$. Of note, none have reported a lower risk of HCC with ETV than TDF. This controversy has motivated many to conduct systematic reviews and meta-analyses. Eleven meta-analyses were published between December 2019 and June 2021 on this controversial issue. The results of the meta-analyses published so far are summarized in Table 1. Although the studies and patients included in the 11 meta-analyses were slightly different, most of the meta-analyses except for two (Tseng et al. ${ }^{[33]}$ and Yuan et al. ${ }^{[34]}$ ) reported that TDF was superior to ETV in preventing HBV-associated HCC when the adjusted HR was pooled. Most meta-analyses performed subgroup analyses and/or metaregression to address between-study heterogeneity. Three major issues were raised during the process of explaining between-study heterogeneity as follows: (1) sample size vs. study setting (i.e., clinical cohort, administrative database, or electronic health record database); (2) cirrhotic subgroup and whether the study included patients with decompensated cirrhosis or not; and (3) difference in follow-up duration between the two drugs.

\section{First issue: sample size vs. study setting}

In the meta-analysis by Choi et al. ${ }^{[35]}$, sample size was one of the most important factors for explaining the between-study heterogeneity in mete-regression analysis. While studies with a larger number of patients showed superiority of TDF in reducing the risk of HCC compared with ETV, studies with a smaller number of patients did not. Another meta-analysis by Tseng et al ${ }^{[33]}$ addressed this issue by observing the differences in the study setting. TDF showed a similar risk for HCC among hospital-based cohort studies $(\mathrm{HR}=1.03$; 95\%CI: 0.88-1.21), whereas TDF was associated with a lower HCC risk among studies based on the administrative claims database $\left(\mathrm{aHR}=0.67 ; 95 \% \mathrm{CI}\right.$ : 0.59-0.76 ${ }^{[33]}$. Tseng et al. ${ }^{[33]}$ explained these discrepant results between clinical cohorts and administrative database studies by the difference in residual 
Table 1. Summary of meta-analyses comparing the risk of HCC between ETV and TDF

\begin{tabular}{|c|c|c|c|c|c|}
\hline Authors & $\begin{array}{l}\text { No. of } \\
\text { studies }\end{array}$ & $\begin{array}{l}\text { No. of } \\
\text { patients }\end{array}$ & $\begin{array}{l}\text { Unadjusted HR } \\
(95 \% \mathrm{Cl})\end{array}$ & $\begin{array}{l}\text { Adjusted HR } \\
(95 \% \mathrm{Cl})\end{array}$ & $\begin{array}{l}\text { Explanation for } \\
\text { heterogeneity }\end{array}$ \\
\hline Zhang et al. ${ }^{[51]}$ & 7 & 3698 & $0.66(0.49-0.89)^{a}$ & NA & No \\
\hline Li et al. ${ }^{[40]}$ & 32 & 78,136 & $0.87(0.73-1.04)^{\mathrm{a}}$ & NA & Subgroup analyses \\
\hline Gu et al..$^{[37]}$ & 11 & 70,864 & $0.75(0.65-0.87)$ & $0.71(0.63-0.79)$ & Subgroup analyses \\
\hline Wang et al. ${ }^{[52]}$ & 8 & 4814 & $0.66(0.41-1.05)^{a}$ & NA & No \\
\hline Dave et al. ${ }^{[53]}$ & 14 & 69,336 & $0.66(0.41-1.05)^{a}$ & $0.79(0.63-0.99)$ & Meta-regression \\
\hline Choi et al. ${ }^{[35]}$ & 15 & 61,787 & $0.80(0.69-0.93)$ & $0.75(0.58-0.97)$ & Meta-regression \\
\hline Liu et al. ${ }^{[54]}$ & 7 & 35,785 & NA & $0.75(0.58-0.96)$ & No \\
\hline Tseng et al. ${ }^{[33]}$ & 15 & 88,141 & $0.75(0.54-1.03)$ & $0.88(0.73-1.07)$ & Subgroup analyses \\
\hline Cheung et al. ${ }^{[38]}$ & 13 & 85,008 & NA & $0.81(0.67-0.99)$ & Subgroup analyses \\
\hline Yuan et al. ${ }^{[34]}$ & 13 & 80,202 & $0.75(0.60-0.95)$ & $0.86(0.72-1.04)$ & Subgroup analyses \\
\hline Jeong et al. ${ }^{[39]}$ & 16 & 50,595 & $0.63(0.43-0.93)^{\mathrm{a}}$ & $0.74(0.63-0.87)$ & Subgroup analyses \\
\hline
\end{tabular}

${ }^{a}$ These meta-analyses reported risk ratio or rate ratio instead of HR. CI: Confidence interval; ETV: entecavir; HCC: hepatocellular carcinoma; HR: hazard ratio; NA: not applicable; TDF: tenofovir disoproxil fumarate.

confounding as the information contained in administrative databases is less precise and prone to errors because of inaccurate coding. However, this may be an overly simplified explanation. Determining the optimal sample size for a clinical study is critical to assure an adequate power to detect a clinical significance. To achieve a power of 0.8 for a type I error level of 0.05 with an assumed HR of 0.88 as reported in Tseng et al. ${ }^{[33]}, 36,364$ patients would be required. Thus, studies that concluded that HCC risks were similar between the two drugs are subject to a type II error. Showing non-significance in the outcome between the two drugs is a lot easier than finding significant differences in comparative effectiveness research $^{[36]}$. Considering that retrospective studies, particularly with a small sample size, entail numerous confounding factors, simply pooling the HR in a meta-analysis is part of the solution for addressing the conflict issue, but it is certainly not the complete solution.

\section{Second issue: presence and stage of cirrhosis}

Subgroup analyses were conducted in most of the meta-analyses to explain the between-study heterogeneity. In a subgroup analysis based on cirrhosis status, TDF was consistently associated with a lower risk of HCC compared with ETV in CHB patients with cirrhosis ${ }^{[3,35,37-39]}$. Moreover, another important determinant of the between-study heterogeneity was whether the study included patients with decompensated cirrhosis or not as shown in a meta-regression analysis by Choi et al.$^{[35]}$. TDF showed a significantly lower HCC risk over ETV ( $\mathrm{HR}=0.69 ; 95 \% \mathrm{CI}$ : 0.55-0.85; $P<0.001)$ in studies including patients with decompensated cirrhosis, whereas no difference between the two drugs (HR = 0.90; 95\%CI: 0.76-1.06; $P$ $=0.20$ ) was found in studies excluding those patients ${ }^{[35]}$. In Korea where the most studies regarding this issue were performed, the landmark study by Choi et al. ${ }^{[13]}$ that showed the superiority of TDF included patients with decompensated cirrhosis, whereas the other studies with a relatively large sample size showing similar risk of HCC between the two drugs did not, which may explain the inconsistent results among studies ${ }^{[16,17]}$. It is well-known that patients with cirrhosis, especially decompensated cirrhosis, have a very high risk of developing HCC. Considering that high case numbers prevent the occurrence of a type II error and facilitate statistically meaningful conclusions being made, the difference in effectiveness between the two drugs in cirrhotic subgroup or studies including patients with decompensated cirrhosis could represent a better preventive effect of TDF. 


\section{Third issue: differences in the follow-up time}

Because ETV was introduced earlier than TDF, ETV-treated patients had longer follow-up durations in most studies. In the study by Lee et al. ${ }^{[17]}$, for example, the median follow-up duration was longer in patients treated with ETV than in those treated with TDF (60 months vs. 36.4 months, respectively). The difference in the follow-up duration due to the asynchronous introduction of the two treatments may cause a bias in either direction. Previous meta-analyses by Tseng et al. ${ }^{[33]}$ and Li et al. ${ }^{[40]}$ also noticed this point, and they performed subgroup analyses based on follow-up duration. In studies with a follow-up duration of ETV that was longer than TDF by more than one year, TDF was significantly associated with a lower risk of HCC $(\mathrm{aHR}=0.69 ; 95 \% \mathrm{CI}: 0.61-0.79)$. However, there was no difference in the risk of HCC between the two treatments in studies with a minimal disparity of less than one year in the follow-up duration $(\mathrm{aHR}=0.88$; 95\%CI: 0.70-1.11) $)^{[33]}$. The patient warehousing phenomenon, which indicates postponing treatment until a new effective drug becomes available, may have contributed to the bias toward prescribing ETV to sicker and older patients before TDF became available. However, it should be noted that TDF consistently showed a superiority over ETV ( $\mathrm{HR}=0.75$; 95\% CI: 0.58-0.97) in pooled analysis of PS-matched cohorts from available studies in which the baseline characteristics were well balanced ${ }^{[35]}$.

Furthermore, patients with favorable outcomes such as HBeAg loss, HBsAg loss, and fibrosis/cirrhosis regression are more likely to be enriched in the longer-term follow-up cohort ${ }^{[41,42]}$, which results in attrition bias acting in favor of ETV. In addition, it is well-known that the preventive effect of antiviral treatment against HCC becomes increasingly evident over time ${ }^{[1,43,44]}$. Thus, it is unlikely that a longer follow-up duration of ETV may be biased in favor of TDF.

From a statistical perspective, all published studies calculated HR with a Cox proportional hazards model, which means that the proportional hazard assumption was satisfied in all studies and the HR comparing TDF and ETV was constant with respect to time. This implies that the issue of the difference in the followup time between the two drugs may not be statistically important.

\section{PLAUSIBLE EXPLANATIONS FOR THE SUPERIORITY OF TDF}

The mechanism underlying the reduced HCC risk associated with TDF over ETV is unclear. It has been postulated that antiviral treatment reduces the HCC risk by modifying three components on a different time scale $^{[45]}$. Liver injury or inflammation are the first to be affected as antiviral treatment suppresses viral replication. Second, regression of fibrosis/cirrhosis due to long-term antiviral treatment may substantially reduce the risk of HCC. Lastly, the risk of HCC may further decrease when antiviral treatment prevents the formation of cccDNA and subsequent host-genome integration ${ }^{[45]}$. Based on this postulation, several plausible explanations could be noted. TDF has a higher antiviral potency, which may affect all of the subsequent modifiable components. In a study by Choi et al. ${ }^{[13]}$, at one year of treatment, TDF treatment resulted in higher rates of virological response and ALT normalization. Yip et al. ${ }^{[14]}$ also observed a higher rate of virological response of TDF compared with ETV at one year of treatment. This was consistent with the findings of recent meta-analyses, which found that the virological response was higher with TDF compared with ETV ${ }^{[37,46]}$. In a small randomized controlled trial comparing the efficacy of TDF and ETV, patients treated with TDF had a greater reduction in their HBsAg level than those treated with ETV ${ }^{[47]}$. Moreover, Papatheodoridis et al ${ }^{[18]}$ found more frequent elastographic reversion of cirrhosis (liver stiffness measurements of $\geq 12 \mathrm{kPa}$ ) in TDF compared with ETV (73.8\%vs. 61.5\%; $P=0.038$ ) after five years of treatment despite similar rates of HCC development between the two treatments. This may suggest that TDF is better at modifying the second modifiable component (i.e., fibrosis or cirrhosis). In addition, a translational research study found a higher interferon lambda-3 level, which had a potent antitumor activity against $\mathrm{HCC}^{[48,49]}$, in patients treated with TDF compared with those treated with $\mathrm{ETV}^{[50]}$. 


\section{CONCLUSION}

Since the study by Choi et al. ${ }^{[13]}$ first reported a better preventive effect of TDF than ETV against HCC, many studies have shown no difference between the two drugs, an issue that has become a matter of contention in the field of hepatology. Nevertheless, all of the studies comparing the risk of HCC between ETV and TDF were either neutral or in favor of TDF. No study was in favor of ETV over TDF. In line with observational studies showing one direction favoring TDF or no direction, most meta-analyses published thus far have also reported the superiority of TDF over ETV in preventing HCC. However, a large degree of uncertainty remains for this issue due to a lack of randomized clinical trials comparing the preventive efficacy of ETV and TDF against HCC occurrence. Bias remains in many observational studies due to a lack of some confounding factors such as presence of comorbid diseases and family history of HCC. Considering the inconsistent methodologies of the previous observational studies, simply pooling the estimates in a meta-analysis is not a definitive answer to this issue. Moreover, a randomized clinical trial would not be feasible in the near future given the need for a large number of patients with a sufficient length of follow-up time. Thus, an alternative approach such as an individual patient data meta-analysis, which allows for adjusting bias with consistent methodologies across all datasets, should be taken into consideration. This would provide a more robust estimate of HCC risk between ETV and TDF in patients with CHB and help to identify the subgroup of patients who will have more benefit from TDF than ETV.

\section{DECLARATIONS}

\section{Authors' contributions}

Wrote the entire manuscript and designed figure and table: Choi WM

Overviewed and edited the entire manuscript: Choi J

\section{Availability of data and materials}

Not applicable.

\section{Financial support and sponsorship}

This work was supported by The Research Supporting Program of The Korean Association for the Study of the Liver and The Korean Liver Foundation.

\section{Conflicts of interest}

Both authors declared that there are no conflicts of interest.

\section{Ethical approval and consent to participate}

Not applicable.

\section{Consent for publication}

Not applicable.

\section{Copyright}

(c) The Author(s) 2021.

\section{REFERENCES}

1. Schweitzer A, Horn J, Mikolajczyk RT, Krause G, Ott JJ. Estimations of worldwide prevalence of chronic hepatitis B virus infection: a systematic review of data published between 1965 and 2013. Lancet 2015;386:1546-55. DOI PubMed

2. Razavi-shearer D, Gamkrelidze I, Nguyen MH, et al. Global prevalence, treatment, and prevention of hepatitis B virus infection in 2016: a modelling study. Lancet Gastroenterol Hepatol 2018;3:383-403. DOI PubMed

3. Vos T, Abajobir AA, Abate KH, et al. Global, regional, and national incidence, prevalence, and years lived with disability for 328 diseases and injuries for 195 countries, 1990-2016: a systematic analysis for the Global Burden of Disease Study 2016. Lancet 2017;390:1211-59. DOI PubMed PMC

4. Llovet JM, Kelley RK, Villanueva A, et al. Hepatocellular carcinoma. Nat Rev Dis Primers 2021;7:6. DOI PubMed 
5. Sepanlou SG, Safiri S, Bisignano C, et al. The global, regional, and national burden of cirrhosis by cause in 195 countries and territories, 1990-2017: a systematic analysis for the Global Burden of Disease Study 2017. Lancet Gastroenterol Hepatol 2020;5:24566. DOI PubMed PMC

6. Chen CJ, Yang HI, Su J, et al; REVEAL-HBV Study Group. Risk of hepatocellular carcinoma across a biological gradient of serum hepatitis B virus DNA level. JAMA 2006;295:65-73. DOI PubMed

7. Association for the Study of the Liver (KASL). KASL clinical practice guidelines for management of chronic hepatitis B. Clin Mol Hepatol 2019;25:93-159. DOI

8. Association for the Study of the Liver, European Association for the Study of the Liver. EASL 2017 Clinical Practice Guidelines on the management of hepatitis B virus infection. J Hepatol 2017;67:370-98. DOI

9. Terrault NA, Lok ASF, McMahon BJ, et al. Update on prevention, diagnosis, and treatment of chronic hepatitis B: AASLD 2018 hepatitis B guidance. Hepatology 2018;67:1560-99. DOI PubMed PMC

10. Sarin SK, Kumar M, Lau GK, et al. Asian-Pacific clinical practice guidelines on the management of hepatitis B: a 2015 update. Hepatol Int 2016;10:1-98. DOI PubMed PMC

11. Ahn J, Lim JK, Lee HM, et al. Lower observed hepatocellular carcinoma incidence in chronic hepatitis B patients treated with entecavir: results of the ENUMERATE Study. Am J Gastroenterol 2016;111:1297-304. DOI PubMed

12. Liu K, Choi J, Le A, et al. Tenofovir disoproxil fumarate reduces hepatocellular carcinoma, decompensation and death in chronic hepatitis B patients with cirrhosis. Aliment Pharmacol Ther 2019;50:1037-48. DOI PubMed

13. Choi J, Kim HJ, Lee J, Cho S, Ko MJ, Lim YS. Risk of hepatocellular carcinoma in patients treated with entecavir vs tenofovir for chronic hepatitis B: a korean nationwide cohort study. JAMA Oncol 2019;5:30-6. DOI PubMed PMC

14. Yip TC, Wong VW, Chan HL, Tse YK, Lui GC, Wong GL. Tenofovir is associated with lower risk of hepatocellular carcinoma than entecavir in patients with chronic HBV infection in China. Gastroenterology 2020;158:215-25.e6. DOI PubMed

15. Ha Y, Chon YE, Kim MN, Lee JH, Hwang SG. Hepatocellular carcinoma and death and transplantation in chronic hepatitis B treated with entecavir or tenofovir disoproxil fumarate. Sci Rep 2020;10:13537. DOI PubMed PMC

16. Kim SU, Seo YS, Lee HA, et al. A multicenter study of entecavir vs. tenofovir on prognosis of treatment-naïve chronic hepatitis B in South Korea. J Hepatol 2019;71:456-64. DOI PubMed

17. Lee SW, Kwon JH, Lee HL, et al. Comparison of tenofovir and entecavir on the risk of hepatocellular carcinoma and mortality in treatment-naïve patients with chronic hepatitis B in Korea: a large-scale, propensity score analysis. Gut 2020;69:1301-8. DOI PubMed PMC

18. Papatheodoridis GV, Dalekos GN, Idilman R, et al. Similar risk of hepatocellular carcinoma during long-term entecavir or tenofovir therapy in Caucasian patients with chronic hepatitis B. J Hepatol 2020;73:1037-45. DOI PubMed

19. Chen $\mathrm{CH}$, Chen $\mathrm{CY}$, Wang JH, et al. Comparison of incidence of hepatocellular carcinoma between chronic hepatitis B patients with cirrhosis treated with entecavir or tenofovir in Taiwan - a retrospective study. Am J Cancer Res 2020;10:3882-95. PubMed PMC

20. Kim R, Telep L, Lu M, et al. IDDF2020-ABS-0057 risk of incident hepatocellular carcinoma (HCC) in chronic hepatitis B (CHB) virus-infected patients treated with tenofovir disoproxil fumarate (TDF) versus entecavir (ETV): a US administrative claims analysis. Gut 2020;69:A73. DOI

21. Zhang M, Wang D, Liu H, Li H. Tenofovir decrease hepatocellular carcinoma recurrence in chronic hepatitis B patients after liver resection. Infect Agent Cancer 2018;13:19. DOI PubMed PMC

22. Ge Z, Ma J, Qiao B, Wang Y, Zhang H, Gou W. Impact of tenofovir antiviral treatment on survival of chronic hepatitis B related hepatocellular carcinoma after hepatectomy in Chinese individuals from Qingdao municipality. Medicine (Baltimore) 2020;99:e21454. DOI PubMed PMC

23. Choi J, Jo C, Lim YS. Tenofovir versus entecavir on recurrence of hepatitis B virus-related hepatocellular carcinoma after surgical resection. Hepatology 2021;73:661-73. DOI PubMed

24. Imamura H, Matsuyama Y, Tanaka E, et al. Risk factors contributing to early and late phase intrahepatic recurrence of hepatocellular carcinoma after hepatectomy. J Hepatol 2003;38:200-7. DOI PubMed

25. Chen YJ, Yeh SH, Chen JT, et al. Chromosomal changes and clonality relationship between primary and recurrent hepatocellular carcinoma. Gastroenterology 2000;119:431-40. DOI PubMed

26. Chang TS, Yang YH, Chen WM, et al. Long-term risk of primary liver cancers in entecavir versus tenofovir treatment for chronic hepatitis B. Sci Rep 2021;11:1365. DOI PubMed PMC

27. Ha I, Chung JW, Jang ES, Jeong SH, Kim JW. Comparison of the on-treatment risks for hepatocellular carcinoma between entecavir and tenofovir: a propensity score matching analysis. J Gastroenterol Hepatol 2020;35:1774-81. DOI PubMed

28. Shin JW, Jeong J, Jung SW, et al. Comparable incidence of hepatocellular carcinoma in chronic hepatitis B Patients treated with entecavir or tenofovir. Dig Dis Sci 2021;66:1739-50. DOI PubMed

29. Hsu YC, Wong GL, Chen CH, et al. Tenofovir versus entecavir for hepatocellular carcinoma prevention in an international consortium of chronic hepatitis B. Am J Gastroenterol 2020;115:271-80. DOI PubMed

30. Oh H, Yoon EL, Jun DW, et al; Long-Term Safety of Entecavir and Tenofovir in Patients With Treatment-Naive Chronic Hepatitis B Virus (CHB) Infection (SAINT) Study. No difference in incidence of hepatocellular carcinoma in patients with chronic hepatitis B virus infection treated with entecavir vs tenofovir. Clin Gastroenterol Hepatol 2020;18:2793-802.e6. DOI PubMed

31. S; ANRS/AFEF study group. Similar 5-year HCC occurrence in Tenofovir- and Entecavir-treated HBV chronic infection in the French AFEF/ANRS CO22 Hepather cohort. Aliment Pharmacol Ther 2021;53:616-29. DOI PubMed

32. Su F, Berry K, Ioannou GN. No difference in hepatocellular carcinoma risk between chronic hepatitis B patients treated with entecavir versus tenofovir. Gut 2021;70:370-8. DOI PubMed 
33. Tseng C, Hsu Y, Chen T, et al. Hepatocellular carcinoma incidence with tenofovir versus entecavir in chronic hepatitis B: a systematic review and meta-analysis. Lancet Gastroenterol Hepatol 2020;5:1039-52. DOI PubMed

34. Yuan J, Peng Y, Hao FB, Wang YQ, Wang CR, Zhong GC. No difference in hepatocellular carcinoma risk in chronic hepatitis B patients treated with tenofovir vs entecavir: evidence from an updated meta-analysis. Aging (Albany NY) 2021;13:7147-65. DOI PubMed PMC

35. Choi WM, Choi J, Lim YS. Effects of tenofovir vs entecavir on risk of hepatocellular carcinoma in patients with chronic HBV infection: a systematic review and meta-analysis. Clin Gastroenterol Hepatol 2021;19:246-58.e9. DOI PubMed

36. Flemming JA, Terrault NA. Tenofovir vs entecavir for hepatocellular carcinoma prevention in patients with chronic hepatitis B: one of these things is not like the other. JAMA Oncol 2019;5:17-8. DOI

37. Gu L, Yao Q, Shen Z, et al. Comparison of tenofovir versus entecavir on reducing incidence of hepatocellular carcinoma in chronic hepatitis B patients: a systematic review and meta-analysis. J Gastroenterol Hepatol 2020;35:1467-76. DOI PubMed

38. Cheung KS, Mak LY, Liu SH, et al. Entecavir vs tenofovir in hepatocellular carcinoma prevention in chronic hepatitis B infection: a systematic review and meta-analysis. Clin Transl Gastroenterol 2020;11:e00236. DOI PubMed PMC

39. Jeong S, Cho Y, Park SM, Kim W. Differential effectiveness of tenofovir and entecavir for prophylaxis of hepatocellular carcinoma in chronic hepatitis B patients depending on coexisting cirrhosis and prior exposure to antiviral therapy: a systematic review and metaanalysis. J Clin Gastroenterol 2021;55:e77-86. DOI PubMed

40. Li M, Lv T, Wu S, et al. Tenofovir versus entecavir in lowering the risk of hepatocellular carcinoma development in patients with chronic hepatitis B: a critical systematic review and meta-analysis. Hepatol Int 2020;14:105-14. DOI PubMed

41. Lam YF, Seto WK, Wong D, et al. Seven-year treatment outcome of entecavir in a real-world cohort: effects on clinical parameters, HBsAg and HBcrAg levels. Clin Transl Gastroenterol 2017;8:e125. DOI PubMed PMC

42. Lim TH, Gane E, Moyes C, Borman B, Cunningham C. Serological and clinical outcomes of horizontally transmitted chronic hepatitis B infection in New Zealand Māori: results from a 28-year follow-up study. Gut 2015;64:966-72. DOI PubMed

43. Papatheodoridis GV, Idilman R, Dalekos GN, et al. The risk of hepatocellular carcinoma decreases after the first 5 years of entecavir or tenofovir in Caucasians with chronic hepatitis B. Hepatology 2017;66:1444-53. DOI PubMed

44. Kim WR, Loomba R, Berg T, et al. Impact of long-term tenofovir disoproxil fumarate on incidence of hepatocellular carcinoma in patients with chronic hepatitis B. Cancer 2015;121:3631-8. DOI PubMed

45. Udompap P, Kim WR. Development of Hepatocellular Carcinoma in Patients With Suppressed Viral Replication: Changes in Risk Over Time. Clin Liver Dis (Hoboken) 2020;15:85-90. DOI PubMed PMC

46. Chen MB, Wang $\mathrm{H}$, Zheng $\mathrm{QH}$, et al. Comparative efficacy of tenofovir and entecavir in nucleos(t)ide analogue-naive chronic hepatitis B: a systematic review and meta-analysis. PLoS One 2019;14:e0224773. DOI PubMed PMC

47. Koike K, Suyama K, Ito H, Itoh H, Sugiura W. Randomized prospective study showing the non-inferiority of tenofovir to entecavir in treatment-naïve chronic hepatitis B patients. Hepatol Res 2018;48:59-68. DOI PubMed

48. Abushahba W, Balan M, Castaneda I, et al. Antitumor activity of type I and type III interferons in BNL hepatoma model. Cancer Immunol Immunother 2010;59:1059-71. DOI PubMed PMC

49. Sato A, Ohtsuki M, Hata M, Kobayashi E, Murakami T. Antitumor activity of IFN-lambda in murine tumor models. J Immunol 2006;176:7686-94. DOI PubMed

50. Murata K, Asano M, Matsumoto A, et al. Induction of IFN- $\lambda 3$ as an additional effect of nucleotide, not nucleoside, analogues: a new potential target for HBV infection. Gut 2018;67:362-71. DOI

51. Zhang Z, Zhou Y, Yang J, Hu K, Huang Y. The effectiveness of TDF versus ETV on incidence of HCC in CHB patients: a meta analysis. BMC Cancer 2019;19:511. DOI PubMed PMC

52. Wang X, Liu X, Dang Z, et al. Nucleos(t)ide analogues for reducing hepatocellular carcinoma in chronic hepatitis B patients: a systematic review and meta-analysis. Gut Liver 2020;14:232-47. DOI PubMed PMC

53. Dave S, Park S, Murad MH, et al. Comparative effectiveness of entecavir versus tenofovir for preventing hepatocellular carcinoma in patients with chronic hepatitis B: a systematic review and meta-analysis. Hepatology 2021;73:68-78. DOI PubMed PMC

54. Liu H, Shi Y, Hayden JC, Ryan PM, Rahmani J, Yu G. Tenofovir treatment has lower risk of hepatocellular carcinoma than entecavir treatment in patients with chronic hepatitis B: a systematic review and meta-analysis. Liver Cancer 2020;9:468-76. DOI PubMed PMC 